\title{
Multiphasic analysis of whole exome sequencing data identifies a novel mutation of ACTG1 in a nonsyndromic hearing loss family
}

\author{
Gibeom Park ${ }^{1 \dagger}$, Jungsoo Gim ${ }^{3+}$, Ah Reum Kim², Kyu-Hee Han², Hyo-Sang Kim², Seung-Ha Oh², Taesung Park ${ }^{3,4}$,
} Woong-Yang Park ${ }^{6,7^{*}}$ and Byung Yoon Choi ${ }^{2,5^{*}}$

\begin{abstract}
Background: The genetic heterogeneity of sensorineural hearing loss is a major hurdle to the efficient discovery of disease-causing genes. We designed a multiphasic analysis of copy number variation (CNV), linkage, and single nucleotide variation (SNV) of whole exome sequencing (WES) data for the efficient discovery of mutations causing nonsyndromic hearing loss (NSHL).

Results: From WES data, we identified five distinct CNV loci from a NSHL family, but they were not co-segregated among patients. Linkage analysis based on SNVs identified six candidate loci (logarithm of odds [LOD] >1.5). We selected 15 SNVs that co-segregated with NSHL in the family, which were located in six linkage candidate loci. Finally, the novel variant p.M305T in ACTG1 (DFNA20/26) was selected as a disease-causing variant.

Conclusions: Here, we present a multiphasic CNV, linkage, and SNV analysis of WES data for the identification of a candidate mutation causing NSHL. Our stepwise, multiphasic approach enabled us to expedite the discovery of disease-causing variants from a large number of patient variants.
\end{abstract}

Keywords: Hearing loss, Copy number variation, Linkage analysis, Single nucleotide variation, Mutation analysis

\section{Background}

By virtue of the recent development of massively parallel DNA sequencing technologies, access to genomic composition has become easier than ever. With the advantage of exome sequencing, many studies have identified causal variants responsible for numerous disorders. Exome sequencing provides a particularly powerful method with which to identify disease-causing single nucleotide variations (SNVs) in Mendelian disorders [1-4]. Though whole exome sequencing (WES) has been used to successfully discover many genes that cause Mendelian disorders, analysis of WES data remains challenging [1]. An individual exome has more than 20,000 variants compared with the reference genome. Even in familial Mendelian disorders,

\footnotetext{
* Correspondence: woongyang.park@samsung.com; choiby@snubh.org ${ }^{\dagger}$ Equal contributors

${ }^{6}$ Department of Molecular Cell Biology, Sungkyunkwan University School of Medicine, Seoul 135-710, Korea

${ }^{2}$ Department of Otolaryngology, Seoul National University College of Medicine, Seoul 110-799, Korea

Full list of author information is available at the end of the article
}

the overall success rate for identifying disease-causing genes is around 50\% [5]. Thus, many of the potential reasons for failure in the WES approach need to be solved fully in order to realize the promise of WES for routine diagnosis of Mendelian disorders.

Filtering patient data against normal populations and inferring identity-by-descent (IBD) in family studies can enrich candidate genes $[4,6]$. Genetic linkage analysis has also been a powerful tool for isolating potential causal candidate variants. A two-step approach of linkage analysis using single nucleotide polymorphism (SNP) microarrays to detect high logarithm of odds (LOD) score regions and subsequent targeted re-sequencing of regions of interest has been utilized in many genomic studies to intensify the power of detection [7]. Classically, microsatellite markers have been used for linkage analysis, and now millions of dimorphic SNP markers can be used to provide higher resolution in order to pinpoint candidate loci [8]. Currently, there are many efforts to use coding SNP information from WES data to facilitate genetic linkage mapping.

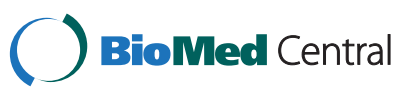

(c) 2013 Park et al.; licensee BioMed Central Ltd. This is an Open Access article distributed under the terms of the Creative Commons Attribution License (http://creativecommons.org/licenses/by/2.0), which permits unrestricted use, distribution, and reproduction in any medium, provided the original work is properly cited. 
Specifically, coding SNP data from WES can be used to establish multiphasic exome analyses based on linkages and SNVs $[4,9]$.

Copy number variation (CNV) has been implicated in both Mendelian diseases [10] and common diseases such as obesity [11] and schizophrenia [12]. The presence of large insertions or deletions in patients is typically investigated prior to SNV analysis by karyotyping, fluorescence in situ hybridization (FISH), and/or array comparative genome hybridization $(\mathrm{aCGH})$. Estimation of $\mathrm{CNV}$ is a challenging aspect of WES analysis, in which local depths of coverage must be mapped to copy numbers. Indeed, aCGH has limitations in detecting high CNV regions. Conversely, CNV data based on WES provides more accurate copy numbers because the depths of exon coverage from WES data vary linearly with real copy numbers [13]. Bioinformatics tools to analyze copy numbers from WES data are now publicly available [14].

Nonsyndromic hearing loss (NSHL) contributes to more than $70 \%$ of inherited cases of hearing loss. To date, approximately 50 genes have been shown to be causally related to NSHL. Many studies have identified more than 129 loci responsible for NSHL; however, 47 loci have not yet been mapped to proper genes $[15,16]$. The complexity of the auditory system may explain why so many genes and loci are linked to hearing loss. The genetic causes of hearing loss can be detected by sequence analysis, which helps clinicians and patients to delineate the basis of the disease. Given that hearing loss in early childhood can affect linguistic development [16], it is important to improve current techniques for identifying genetic alterations that cause NSHL. Earlier identification of such alterations in patients and families may allow for better clinical management of NSHL.

Analysis of WES data can be expanded to obtain more information useful for identifying causative mutations in Mendelian diseases. In this paper, in order to analyze WES data from an entire family, we applied three different methods, namely, $\mathrm{CNV}$, linkage, and segregation analysis. By combining the results obtained from these methods, we efficiently identified a causative mutation from the family data. We applied this approach to WES data from a NSHL family to identify candidate diseasecausing variants.

\section{Results}

\section{Clinical features of a NSHL family}

We identified a Korean family with six members affected by NSHL and seven unaffected members (Figure 1A). Pure tone audiometry (PTA) was performed on nine family members, three of whom (II-2, II-3, and II-5) exhibited profound post-lingual hearing loss. The three members had normal cognitive function and no anomalous-looking features. They went through a battery of clinical tests ranging from general physical examinations, chest X-rays, and simple blood tests to detailed imaging studies including brain MRI and temporal bone CT scans. No abnormalities were detected in the tests, excluding the possibility that hearing loss in these patients was syndromic. The other siblings (II-1, II-7, and II-9) exhibited normal hearing (Figure 1B). Patients II-2, II-3, and II-5 estimated that their hearing loss became severe in their 30's, during which time they started to wear hearing aids. Their hearing loss was further aggravated and became profound in their late forties. Ultimately, patients II- 3 and II- 5 no longer benefited from hearing aids and underwent cochlear implantation. They achieved recognition of common sentences without lip reading one year after implantation. GJB2 is one of the most frequently detected genes in individuals with NSHL, and thus we first investigated the sequence of GJB2 in the NSHL patients. After failing to identify any mutations in GJB2, we performed WES on several members of the Korean family in order to identify a disease-causing mutation.

\section{Copy number analysis using WES data}

WES data was obtained from the parents and six siblings (four affected and four unaffected members, Figure 1A). The mean coverage of each sample ranged from 40.3X to 51.3X, and $87.0 \%$ to $90.5 \%$ of the targeted exome had at least 10 reads. A multiphasic WES analysis was designed to find a causative NSHL mutation (Figure 1C). First, we investigated co-segregation of copy number duplication or deletion in exomes of patients using CONTRA software. We detected five CNV loci with distinct features in the plots (Figure 2A). None of the CNVs co-segregated with affected or unaffected family members. One CNV locus of the CNVs from three members (high copy number exons in II-9, and low copy number exons in II-3 and II-7) was located in 8p23.1, a region that contains beta-defensin genes and SPAG11 (Figure 2B). The following genes were identified as being located at regions of distinct CNVs in the indicated family members: GSTM1 in 1p13.3 (I-1, II-3, II-7, and II-9) (Figure 2C), UGT2B17 in 4q13.2 (I-2 and II-7), BNTL3 in 5q35.3 (II-1), and LILRB2 in 19q13.4 (I-2) (data not shown). We also applied Fisher's exact test for the LOD score per exon to detect co-segregated regions of CNVs, but there were no peaks with values reaching significance. We identified two groups based on the pattern of segregation of SPAG11, GSTM1, and beta-defensin genes to validate the relevance of this method (Figure 2D).

\section{Exome linkage analysis}

Because the pedigree strongly suggested an autosomal dominant mode of inheritance, we identified 17,498 coding autosomal SNVs from WES data and performed single-point linkage analysis. We identified six hot spots where a number of peaks were closely clustered (Figure 3 ). 


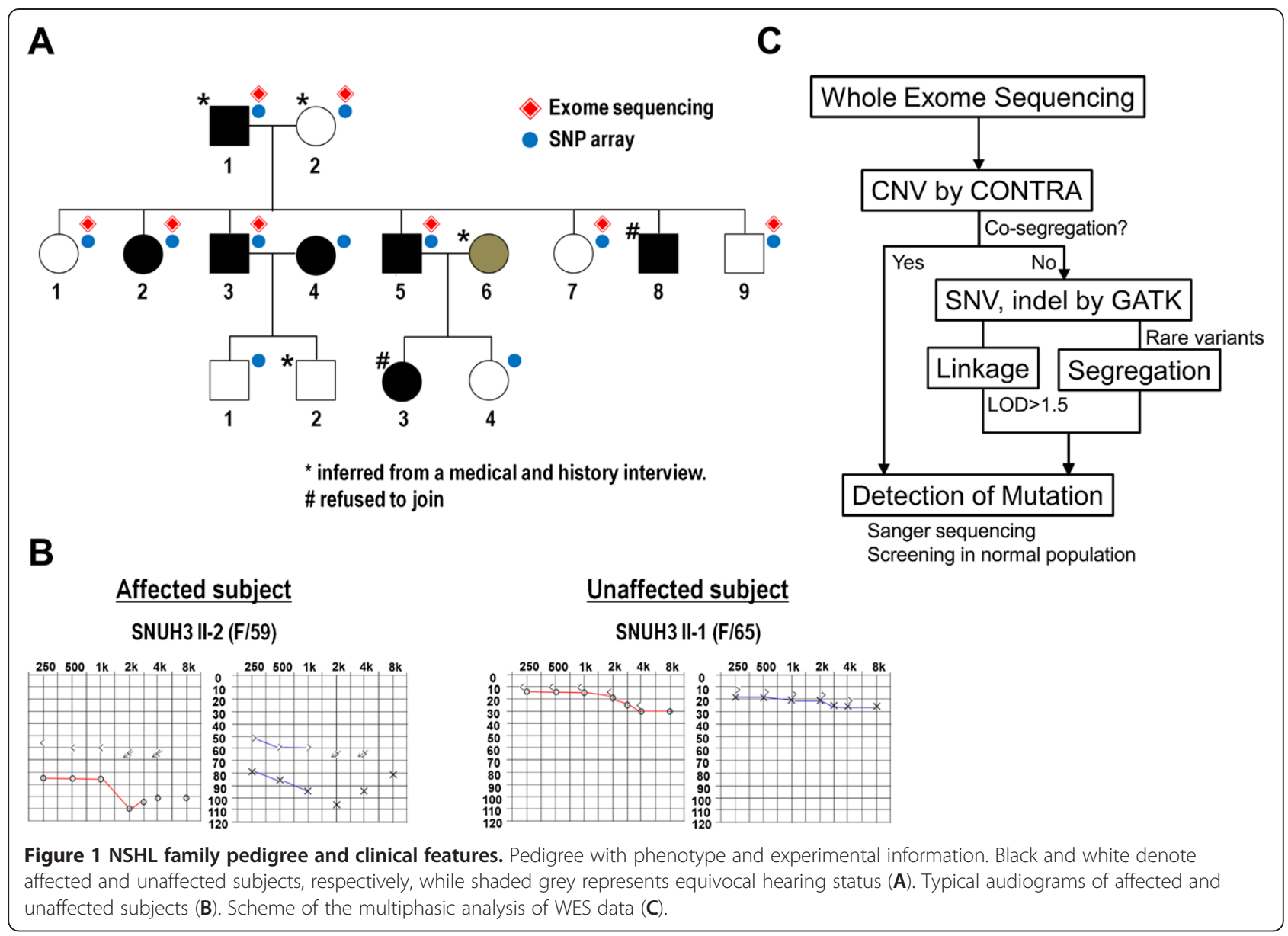

Specifically, we identified peaks on chromosomes 3, 11, $13,14,16$, and 17 consisting of $11,67,2,13,17$, and 13 exons, respectively.

We validated single-point linkages using a SNP microarray containing 328,125 SNPs. Along with the eight initial family members recruited for WES analysis, we included three additional subjects (II-4, III- 1 , and III-4) to validate the significance of peaks obtained from exome linkage analysis. The six hot spots detected from sequencing data were also detected in microarray analysis with a relatively high LOD score (Figure 3). Adding three more subjects to the linkage analysis enhanced the peaks at chromosomes 11 and 17, which consisted of one and three SNPs (LOD score $>2$ ), respectively. The genotype patterns of these four peaks were perfectly matched with an autosomal dominant mode of inheritance.

\section{SNV analysis}

Based on the WES analysis of four affected and four unaffected family members, we identified 18,748 20,025 SNVs and 413 457 indels. These were reduced to 962 1,123 SNVs and 140 153 indels after filtering through the dbSNP135 and 1000 Genome databases. Fifteen variants causing amino acid changes were selected based on their co-segregation pattern within the family (Table 1). All of the 15 variants on chromosomes 3, 11, 13, 16 , and 17 corresponded to regions with high LOD scores (Figure 3). One novel mutation in actin gamma 1 (ACTG1) was identified, consisting of a methionine to threonine substitution at amino acid 305 (p.M305T), This candidate variant was validated by Sanger sequencing and co-segregated with hearing loss in all family members (Figure $4 \mathrm{~A}$ and B).

ACTG1 (DFNA20/26; MIM: 604717) was strictly conserved in 19 of 20 eukaryotes analyzed (HomoloGene:74402), with the M305 codon being conserved in 19 species. Protein damage prediction analysis identified p.M305T as "possibly damaging" by HumDiv, "probably damaging" by HumVar in Polyphen2 [17], and "disease causing" by MutationTaster [18]. The mutation site, Met305, was visualized using the 3D structure of bovine beta-actin bound by adenosine triphosphate (ATP) with profilin (Figure 4C). The methionine was closely located to the ATP molecule. Additionally, Met305 is listed as a predicted residue for the ATP binding site by the Protein Data Bank (PDB). 


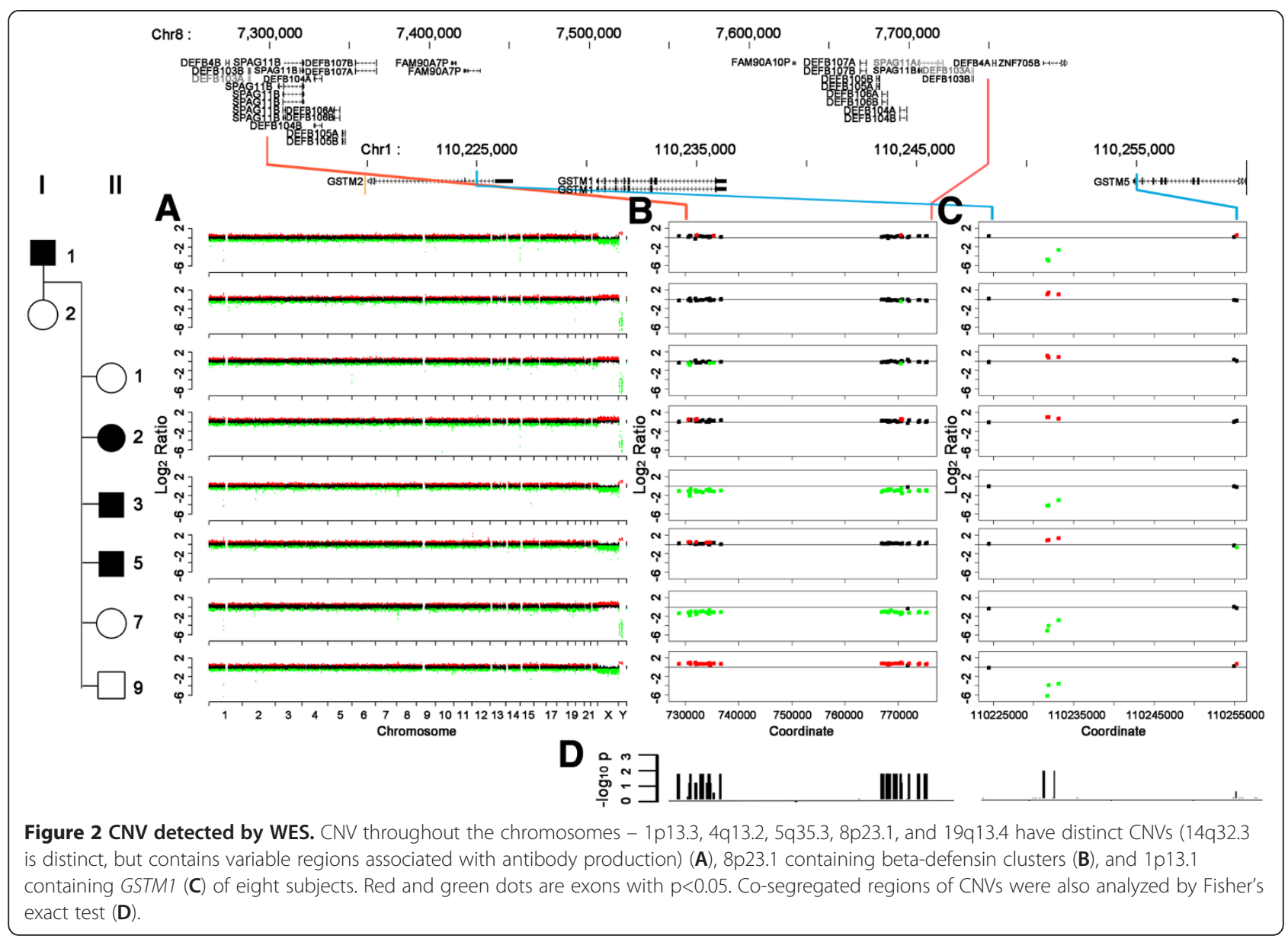

\section{Discussion}

WES is a powerful technique that can be used to discover causative genes in human diseases. Although WES has been integral in identifying more than 1,000 novel genes in Mendelian disorders [1], there is still a need for increased efficiency of gene discovery using WES data. In this regard, we analyzed WES data from a family with a history of NSHL by focusing on three categories of genetic information: CNVs, linkage analysis, and SNVs. Utilizing these data, we undertook a stepwise multiphasic approach to identify disease-causing variations in the family.

8p23.1, which contains a beta-defensin cluster, was detected as a region with high copy number (II-9) and low copy number (II-3 and II-7) (Figure 2). The defensin cluster, containing both alpha- and beta-defensins, was previously studied as a dynamic genomic region with varying copy numbers ranging from one to twelve [19]. The parents had normal copy numbers, which was in contrast to the low copy numbers seen in two children and high copy number observed in one child. A total of four haplotypes of 8p23.1 may have been inherited in this family, and each parent may have had both under- and over-amplified alleles of 8p23.1. The overall copy number of a parent can appear to be normal due to compensation of copy number from over- to under-amplified alleles [20]. In the family in this study, GSTM1 and UGT2B17, genes with frequently reported deletions [21], as well as BNTL3 and LILRB2, exhibited CNVs. We used Fisher's exact test on the affected and unaffected family members after validating this method for 8p23.1 and GSTM1 groups to determine the amplification or deletion of multiple exons that matched the co-segregation pattern of the disease. Multiple statistically significant peaks at 8p23.1 and GSTM1 were identified, and were identical to plots from the first approach. However, there was only one statistically significant peak identified by testing the two groups that segregated with the disease, and this peak did not correlate with disease status. Thus, while WES may provide a method to identify CNV regions with highly similar sequences, determining accurate copy numbers can prove difficult.

Linkage analysis was performed to narrow down the number of candidates based on WES data. Importantly, linkage analysis with a relatively small number of markers still provides useful information. Fewer markers from WES data are available and can be obtained from a SNP microarray, and the markers that are identified may not be evenly 


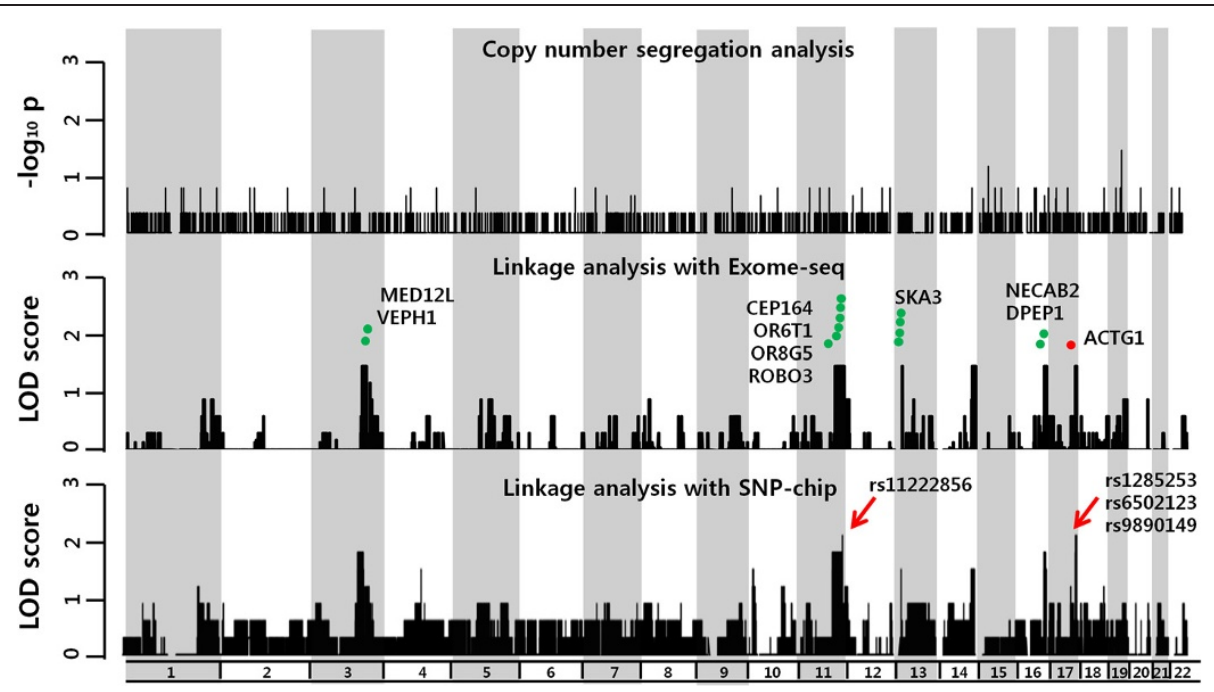

Figure 3 A multiphasic analysis of WES data. WES data were analyzed for exon CNVs and SNVs. Fisher exact test on CNVs detected one exon segregating with NSHL on chr19 (top). Linkage analysis with SNVs called by Exome-seq identified six disease-linked "hot spots" on chr3, chr11, chr13, chr14, chr16, and chr17 (middle). Segregation analysis independently identified 15 SNVs co-segregating with NSHL (green dots). Among them, a novel variant resulting in p.M305T, in ACTG1 on chr17 was validated with Sanger sequencing (red dot). Linkage analysis was also performed with SNP microarray by adding three more subjects in the family. Not only were similar "hot spots" detected, adding more subjects in the analysis enhanced the true peak (red arrow) (bottom).

distributed. Given these limitations, it is necessary to consider the potential disadvantages of this approach. Because we analyzed only exonic SNPs $(\sim 1 \%$ of genome-wide SNPs), we may have lost critical information located outside of exons. In addition, potential genotyping errors in linkage analyses can reduce statistical power for detecting linkage peaks or result in false positive linkage peaks [22]. Even so, the results obtained from the different data sets in this study confirmed the validity of our approach. Linkage analysis requires a large number of subjects to help identify putative loci. Unless a proper number of subjects are available, an informative result is difficult to obtain.

After applying linkage analysis results, the co-segregated variants were all found to be located in the loci of high LOD scores. However, linkage analysis can decrease the number of candidate variants, particularly in instances where candidate variants are widely distributed. Additional linkage analysis of WES data demonstrated a similar

Table 1 Nonsynonymous SNVs and indels identified in patients but not in non-symptomatic family members

\begin{tabular}{|c|c|c|c|c|c|}
\hline Gene & Chr & Nucleotide variation & Amino acid variation & Frequency in 1,000 genome & dbSNP135 \\
\hline MED12L & chr3 & c.G3629A & p.R1210Q & 0.23 & rs3732765 \\
\hline VEPH1 & chr3 & C.T1564C & p.S522P & 0.28 & rs11918974 \\
\hline CWF19L2 & chr11 & c.A2681G & p.Y894C & 0.27 & rs3758911 \\
\hline CEP164 & chr11 & c.G281A & p.S94N & 0.19 & rs490262 \\
\hline OR6T1 & chr11 & c.G465C & p.W155C & 0.0046 & rs150534954 \\
\hline OR8G5 & chr11 & c.G287A & p.C96Y & 0.45 & rs2512168 \\
\hline OR8G5 & chr11 & c.G716A & p.G239E & 0.50 & rs2512167 \\
\hline $\mathrm{ROBO} 3$ & chr11 & c.G1247A & p.R416H & 0.14 & rs3862618 \\
\hline SKA3 & chr13 & C.A1157G & p.K386R & 0.13 & rs11147976 \\
\hline SKA3 & chr13 & c.C1142T & p.T3811 & 0.11 & rs11147977 \\
\hline SKA3 & chr13 & c.G559A & p.V187I & 0.14 & rs61950353 \\
\hline SKA3 & chr13 & c.208delC & p.Q70fs & - & rs151272242 \\
\hline NECAB2 & chr16 & c.C704G & p.T235S & 0.20 & rs2292324 \\
\hline DPEP1 & chr16 & c.G1051C & p.E351Q & 0.24 & rs1126464 \\
\hline ACTG1 & chr17 & c.T914C & p.M305T & - & - \\
\hline
\end{tabular}




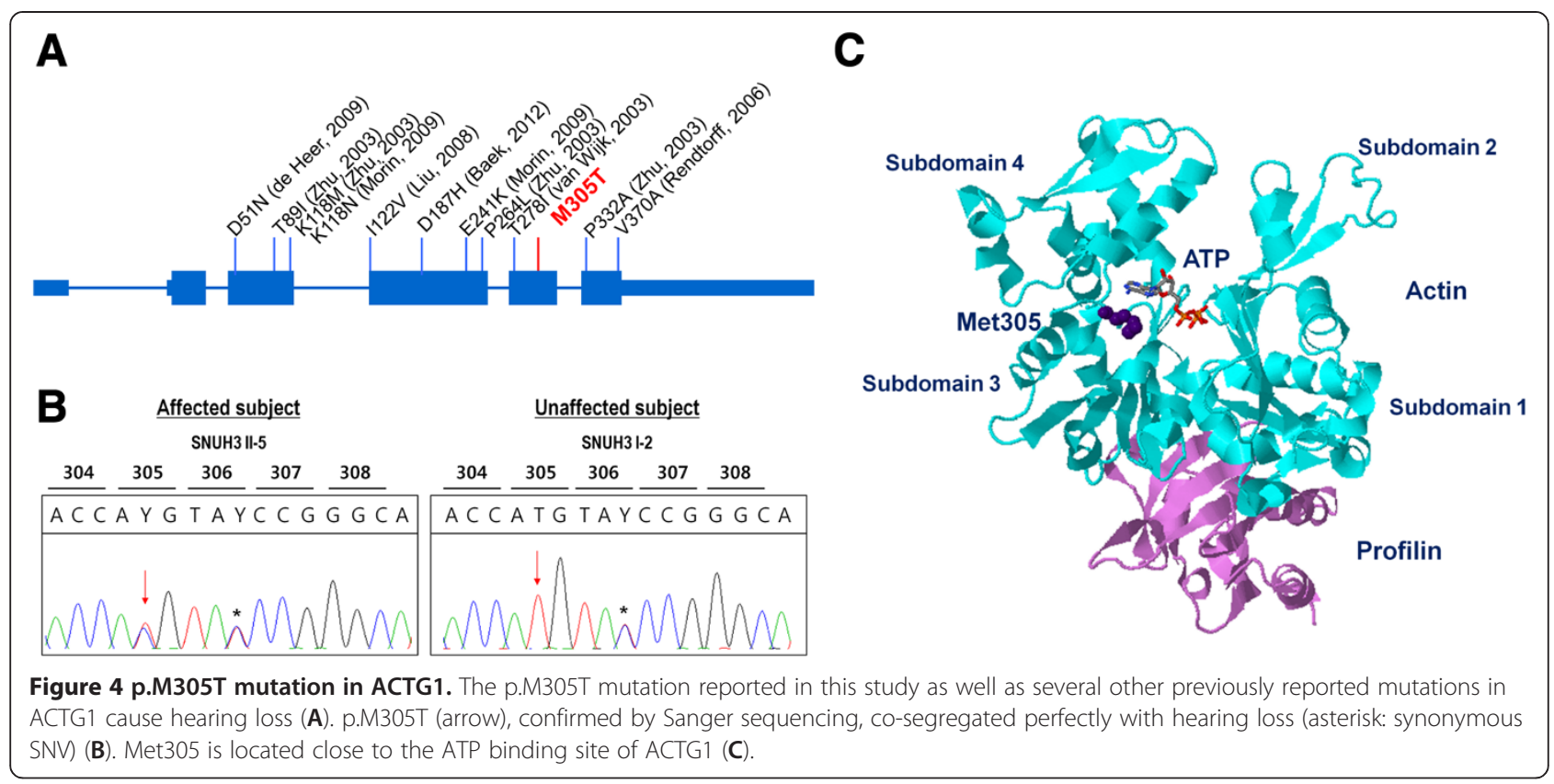

performance to that of SNP microarray data and simultaneously generated results during variant calling. Considering that CNVs could be also detected using this approach, the multiphasic analysis of WES data efficiently narrowed and identified candidate variants and was advantageous compared with established methods such as initial aCGH, variant calling according to WES data alone, or linkage analysis based on SNP microarray data.

Actin is a highly conserved cytoskeletal protein that plays important roles in eukaryotic cell processes such as cell division, migration, endocytosis, and contractility. Actin isoforms are classified into two groups based on expression patterns. ACTA1, ACTA2, ACTC, and ACTG2 are "muscle" actins, predominantly expressed in striated or smooth muscle, whereas ACTB and ACTG1 are cytoplasmic "non-muscle" actins [23]. Autosomal dominant progressive sensorineural hearing loss, DFNA20/26 (MIM: 604717), is caused by a mutation in the gammaactin gene on chromosome 17 at q25.3. Some ACTG1 mutations are associated with Baraitser-Winter syndrome, which is characterized by developmental delay, facial dysmorphologies, brain malformations, colobomas, and variable hearing loss. The constellation of these abnormalities is suggested as the most severe phenotype of ACTG1 mutations [24,25]. A genome-wide screen of DFNA20 localized candidates to $17 \mathrm{q} 25.3$ [26] and mapped the causative missense mutations to highly conserved actin domains of the gamma-actin gene (ACTG1) [27,28]. In vivo and in vitro studies of ACTG1 indicate that it is required for reinforcement and long-term stability of actin filamentous structures of stereocilia, but not for auditory hair cell development, which is in line with the progressive nature of hearing loss related to ACTG1 mutations in humans $[29,30]$. Further, missense mutations in either $A C T B$ or $A C T G 1$ have recently been reported to cause Baraitser-Winter syndrome. Interestingly, of the 11 mutations that cause DFNA20 [27,28,31-34] and 6 mutations that cause Baraitser-Winter syndrome (see OMIM entry *102560) that have been reported, are all missense mutations. The predicted interaction between Met305 and ATP in bovine beta-actin, a protein with a $99 \%$ identity to ACTG1, implies that the mutation of Met305 may influence ATP binding of ACTG1, which is essential for polymerization of G-actin to F-actin.

ACTG1 is predominantly expressed in intestinal epithelial and auditory hair cells [35]. Detection of exclusively missense mutations in this gene may imply that truncating mutations have more severe effects and might cause embryonic lethality. The hearing impaired subjects in this study (II-2, II-3, and II-5) did not report any gastrointestinal complaints. The subjects in this study required cochlear implants, recapitulating what has previously been reported regarding the management of patients with mutations in ACTG1 and resultant NSHL [31]. The severe phenotype and rapid progression of hearing loss to a profound level within one or two decades associated with mutations in ACTG1 necessitates an early molecular genetic diagnosis and timely auditory rehabilitation.

\section{Conclusions}

Two or more platforms (aCGH, SNP array, and WES) have previously been required to generate complex genetic information such as CNVs, linkages, SNVs, and 
indels. In general studies of Mendelian disorders, WES has primarily been utilized to obtain only SNVs and indels. Our study agrees well with other work demonstrating that analysis of WES data also allows for CNV and linkage determination due to its quantitative traits. Given the robust nature of WES data, it is clear that the full capabilities of this relatively new technology have not yet been fully realized. Our multiphasic WES analysis proved very powerful for the interpretation and narrowing of WES results, in particular when a large amount of family data is available.

\section{Methods}

\section{Subjects}

This study was approved by the Institutional Review Boards (IRBs) of Seoul National University Hospital (SNUH) and Seoul National University Bundang Hospital (SNUBH). Written informed consent for participation in the study was obtained from participants or from a parent or guardian in the case of child participants. A three generation pedigree was established for the family (SNUH3) (Figure 1A). Among the 15 subjects in the SNUH3 family, 13 were willing to participate in this genetic study, while two reportedly deaf subjects (II-8 and III-3) refused participation. DNA from blood lymphocytes was isolated from the 12 subjects, while DNA from III-2 was obtained with a buccal swab.

\section{Audiometric analysis}

Pure tone and speech audiometry and physical examinations were performed for nine members of the cohort (Figure 1B). Pure tone audiometry (PTA) with air and bone conduction at frequencies ranging from 250 to $8,000 \mathrm{~Hz}$ was obtained from the recruited subjects according to standard protocols. The auditory phenotype was inferred from thorough medical and developmental history interviews from one deaf subject (I-1), two likely unaffected subjects (I-2 and III-2), and one subject (II-6) with an equivocal hearing status.

\section{WES}

Eight of the 13 recruited subjects (four affected and four unaffected) were chosen for commercial WES (Otogenetics, Norcross, GA) and analyzed as previously reported [3]. Briefly, paired-end reads of $100 \mathrm{bp}$ from the eight subjects were aligned by bwa-0.6.1 to the UCSC hg19 reference genome using default settings. SAMtools and Picards were used to process SAM/BAM files and mark duplicates. Local realignment around indels and base quality score recalibration was done for each sample, and variants were called by a unified genotyper in GATK-1.3. Perl scripts and ANNOVAR were used to annotate variants and search the relevant known SNPs and indels from dbSNP135 and the 1000 Genome database. Variants with a read depth greater than 10 and genotype quality score greater than 30 were filtered for further analysis.

\section{CNV analysis using WES data}

CNVs were detected by CONTRA software [36] using BEDTools to calculate coverage per exon and apply statistics to normalize coverage data and test fold changes. A new baseline file was produced using our data, but we expected to detect distinct deletions or amplifications. Polymerase chain reaction (PCR) duplicates were removed by Picards before using CONTRA.

We tabulated a $3 \times 2$ exon copy variation contingency table based on the whole per-exon CNV status of the eight subjects (Table 2). Fisher's exact test was used to assess the significance of differences between proportions of abnormal copy number events present in affected and unaffected family members. We assumed that all of the subjects were independent in order to conduct an alternative practical method to find loci that segregated with the disease.

\section{Linkage analysis using WES and SNP microarray data}

Using WES data, we filtered out the following variants: those located on sex chromosomes, those with low coverage $(<10 \mathrm{X})$, and those with a low genotype quality score $(<30)$ in any of the eight subjects with 17,498 SNVs. We used a Genome-Wide Human SNP Array 6.0 (Affymetrix, Santa Clara, CA), which contains 328,125 SNP markers located on autosomal chromosomes. We performed parametric linkage analysis with the R package paramlink [37]. The pedigree suggested an autosomal dominant mode of inheritance, and thus we assumed an autosomal dominant model with default values of full penetrance $\left(f_{0}, f_{1}, f_{2}\right)=(0,1,1)$ and disease allele frequency $=1 \mathrm{e}-05$. The penetrance parameters $\mathrm{f}_{0}, \mathrm{f}_{1}$, and $\mathrm{f}_{2}$ were also defined using conventional notation as below.

$$
f_{i}=P(\text { affected } \mid i \text { copies of the disease allele })
$$

The recombination fraction between the disease locus and markers was set to $\theta=0$ by default. We computed single-point LOD scores for all markers. We compared LOD scores from SNP microarray and WES. We matched the subjects and the markers that were common between

Table 2 Exon copy number variation contingency based on the whole per-exon CNV status

\begin{tabular}{cccc}
\hline Copy number & Patient & Normal & Total \\
\hline gain & $n_{21}$ & $n_{20}$ & $n_{2+}$ \\
normal & $n_{11}$ & $n_{10}$ & $n_{1+}$ \\
loss & $n_{01}$ & $n_{00}$ & $n_{0+}$ \\
& $n_{+1}$ & $n_{+0}$ & 8 \\
\hline
\end{tabular}


both platforms using manual python and R scripts. Finally, single-point analyses were performed with all of the data.

\section{D structure of actin gamma-1 (ACTG1)}

Protein damage prediction analysis was performed using HumDiv and HumVar in Polyphen2 [17], and also by MutationTaster [18]. The mutation site was visualized using the 3D structure of bovine beta-actin bound by adenosine triphosphate (ATP) with profilin. Bovine beta-actin actin has a $99 \%$ identity with human gamma-actin. The ATP binding site was analyzed using the Research Collaboratory for Structural Bioinformatics (RCSB) Protein Data Bank (PDB) (www.pdb.org) [38]. PDB entry 2BTF [39] on P60712 (ACTB_BOVIN) with P02584 (PROF1_BOVIN) was downloaded, visualized, and modified by Bioclipse [40] to observe the 305Met residue in 3D.

\section{Competing interests}

The authors declare that they have no competing interests.

\section{Authors' contributions}

GP participated in the design of the study, analysis and interpretation of data; JG analyzed copy number variation from the exome data; AK validated variants by Sanger sequencing and checked segregation of the variant; $\mathrm{KH}$ collected samples from the family; HK analyzed clinical features; SO collected patients and initiated this study; TP have been involved in drafting the manuscript; WP designed the study, wrote the manuscript, revised it; BC participated in the design of the study, interpretation of data, and drafting the manuscript, revising it critically and have given final approval of the version to be published. All authors read and approved the final manuscript.

\section{Acknowledgements}

This study was supported by a grant from the Korea Healthcare Technology R\&D Project, Ministry for Health, Welfare and Family Affairs, Republic of Korea (No. A111377 to B.Y. Choi and No A120017 to W.-Y. Park) and Seoul National University Bundang Hospital research fund (06-2011-124 to B. Y. Choi). The funding agencies had no role in study design, data collection and analysis, decision to publish, or preparation of the manuscript

\section{Author details}

'Departments of Biomedical Sciences, Seoul National University Graduate School, Seoul 110-799, Korea. ${ }^{2}$ Department of Otolaryngology, Seoul National University College of Medicine, Seoul 110-799, Korea. Interdiciplinary Program for Bioinformatics, College of Natural Science, Seoul National University, Seoul 151-742, Korea. ${ }^{4}$ Department of Statistics, College of Natural Science, Seoul National University, Seoul 151-742, Korea. ${ }^{5}$ Department of Otolaryngology, Seoul National University Bundang Hospital, Seongnam 463-707, Korea. ${ }^{6}$ Department of Molecular Cell Biology, Sungkyunkwan University School of Medicine, Seoul 135-710, Korea. Translational Genomics Laboratory, Samsung Genome Institute, Samsung Medical Center, Seoul 135-710, Korea.

Received: 31 October 2012 Accepted: 4 March 2013

Published: 18 March 2013

\section{References}

1. Bamshad MJ, Ng SB, Bigham AW, Tabor HK, Emond MJ, Nickerson DA, Shendure J: Exome sequencing as a tool for Mendelian disease gene discovery. Nat Rev Genet 2011, 12(11):745-755.

2. Yang W, Ahmed M, Elian M, Hady E-SA, Levchenko TS, Sawant RR, Signoretti S, Collins M, Torchilin VP, Goldberg SN: Do Liposomal Apoptotic Enhancers Increase Tumor Coagulation and End-Point Survival in Percutaneous Radiofrequency Ablation of Tumors in a Rat Tumor Model? Radiology 2010, 257(3):685-696.

3. Min BJ, Kim N, Chung T, Kim OH, Nishimura G, Chung CY, Song HR, Kim $H W$, Lee HR, Kim J, et al: Whole-exome sequencing identifies mutations of
KIF22 in spondyloepimetaphyseal dysplasia with joint laxity, leptodactylic type. Am J Hum Genet 2011, 89(6):760-766.

4. Musunuru K, Pirruccello JP, Do R, Peloso GM, Guiducci C, Sougnez C, Garimella KV, Fisher S, Abreu J, Barry AJ, et al: Exome sequencing, ANGPTL3 mutations, and familial combined hypolipidemia. N Eng/ J Med 2010, 363(23):2220-2227.

5. Gilissen C, Hoischen A, Brunner HG, Veltman JA: Disease gene identification strategies for exome sequencing. Eur J Hum Genet 2012, 20(5):490-497.

6. Krawitz PM, Schweiger MR, Rodelsperger C, Marcelis C, Kolsch U, Meisel C, Stephani F, Kinoshita T, Murakami Y, Bauer S, et al: Identity-by-descent filtering of exome sequence data identifies PIGV mutations in hyperphosphatasia mental retardation syndrome. Nat Genet 2010, 42(10):827-829.

7. Cooper GM, Shendure J: Needles in stacks of needles: finding disease-causal variants in a wealth of genomic data. Nat Rev Genet 2011, 12(9):628-640.

8. Nielsen R, Paul JS, Albrechtsen A, Song YS: Genotype and SNP calling from next-generation sequencing data. Nat Rev Genet 2011, 12(6):443-451.

9. Smith KR, Bromhead CJ, Hildebrand MS, Shearer AE, Lockhart PJ, Najmabadi H, Leventer RJ, McGillivray G, Amor DJ, Smith RJ, et al: Reducing the exome search space for Mendelian diseases using genetic linkage analysis of exome genotypes. Genome Biol 2011, 12(9):R85.

10. Cho TJ, Kim OH, Choi IH, Nishimura G, Superti-Furga A, Kim KS, Lee YJ, Park WY: A dominant mesomelic dysplasia associated with a 1.0-Mb microduplication of HOXD gene cluster at 2q31.1. J Med Genet 2010, 47(9):638-639.

11. Sha BY, Yang TL, Zhao LJ, Chen XD, Guo Y, Chen Y, Pan F, Zhang ZX, Dong $\mathrm{SS}, \mathrm{Xu} X \mathrm{H}$, et al: Genome-wide association study suggested copy number variation may be associated with body mass index in the Chinese population. J Hum Genet 2009, 54(4):199-202.

12. Consortium IS: Rare chromosomal deletions and duplications increase risk of schizophrenia. Nature 2008, 455(7210):237-241.

13. Kang GC, Gan AW, Yam A, Tan AB, Tay SC: Mycobacterium abscessus Hand Infections in Immunocompetent Fish Handlers: Case Report. J Hand Surg Am 2010, 35(7):1142-1145.

14. Li J, Lupat R, Amarasinghe KC, Thompson ER, Doyle MA, Ryland GL, Tothill RW, Halgamuge SK, Campbell IG, Gorringe KL: CONTRA: copy number analysis for targeted resequencing. Bioinformatics 2012, 28(10):1307-1313.

15. Hilgert N, Smith RJ, Van Camp G: Forty-six genes causing nonsyndromic hearing impairment: which ones should be analyzed in DNA diagnostics? Mutat Res 2009, 681 (2-3):189-196.

16. Dror AA, Avraham KB: Hearing impairment: a panoply of genes and functions. Neuron 2010, 68(2):293-308.

17. Adzhubei IA, Schmidt S, Peshkin L, Ramensky VE, Gerasimova A, Bork P, Kondrashov AS, Sunyaev SR: A method and server for predicting damaging missense mutations. Nat Methods 2010, 7(4):248-249.

18. Schwarz JM, Rodelsperger C, Schuelke M, Seelow D: MutationTaster evaluates disease-causing potential of sequence alterations. Nat Methods 2010, 7(8):575-576.

19. Hollox EJ, Barber JC, Brookes AJ, Armour JA: Defensins and the dynamic genome: what we can learn from structural variation at human chromosome band 8p23.1. Genome Res 2008, 18(11):1686-1697.

20. Carelle-Calmels N, Saugier-Veber P, Girard-Lemaire F, Rudolf G, Doray B, Guerin E, Kuhn P, Arrive M, Gilch C, Schmitt E, et al: Genetic compensation in a human genomic disorder. N Engl J Med 2009, 360(12):1211-1216.

21. Huang RS, Chen P, Wisel S, Duan S, Zhang W, Cook EH, Das S, Cox NJ, Dolan ME: Population-specific GSTM1 copy number variation. Hum Mol Genet 2009, 18(2):366-372.

22. Cherny SS, Abecasis GR, Cookson WO, Sham PC, Cardon LR: The effect of genotype and pedigree error on linkage analysis: analysis of three asthma genome scans. Genet Epidemiol 2001, 21(Suppl 1):S117-S122.

23. Sonnemann KJ, Fitzsimons DP, Patel JR, Liu Y, Schneider MF, Moss RL, Ervasti JM: Cytoplasmic gamma-actin is not required for skeletal muscle development but its absence leads to a progressive myopathy. Dev Cell 2006, 11(3):387-397.

24. Drummond MC, Belyantseva IA, Friderici KH, Friedman TB: Actin in hair cells and hearing loss. Hear Res 2012, 288(1-2):89-99.

25. Riviere JB, van Bon BW, Hoischen A, Kholmanskikh SS, O'Roak BJ, Gilissen C, Gijsen S, Sullivan CT, Christian SL, Abdul-Rahman OA, et al: De novo mutations in the actin genes ACTB and ACTG1 cause Baraitser-Winter syndrome. Nat Genet 2012, 44(4):440-444. S441-442.

26. Morell RJ, Friderici KH, Wei S, Elfenbein JL, Friedman TB, Fisher RA: A new locus for late-onset, progressive, hereditary hearing loss DFNA20 maps to $17 q 25$. Genomics 2000, 63(1):1-6. 
27. Zhu M, Yang T, Wei S, DeWan AT, Morell RJ, Elfenbein JL, Fisher RA, Leal SM, Smith RJ, Friderici KH: Mutations in the gamma-actin gene (ACTG1) are associated with dominant progressive deafness (DFNA20/26). Am J Hum Genet 2003, 73(5):1082-1091.

28. van Wijk E, Krieger E, Kemperman MH, De Leenheer EM, Huygen PL, Cremers CW, Cremers FP, Kremer H: A mutation in the gamma actin 1 (ACTG1) gene causes autosomal dominant hearing loss (DFNA20/26). $J$ Med Genet 2003, 40(12):879-884.

29. Belyantseva IA, Perrin BJ, Sonnemann KJ, Zhu M, Stepanyan R, McGee J, Frolenkov GI, Walsh EJ, Friderici KH, Friedman TB, et al: Gamma-actin is required for cytoskeletal maintenance but not development. Proc Natl Acad Sci U S A 2009, 106(24):9703-9708.

30. Perrin BJ, Sonnemann KJ, Ervasti JM, Perrin BJ, Sonnemann KJ, Ervasti JM: beta-actin and gamma-actin are each dispensable for auditory hair cell development but required for Stereocilia maintenance. PLoS Genet 2010 6(10):e1001158.

31. Rendtorff ND, Zhu M, Fagerheim T, Antal TL, Jones M, Teslovich TM, Gillanders EM, Barmada M, Teig E, Trent JM, et al: A novel missense mutation in ACTG1 causes dominant deafness in a Norwegian DFNA20/ 26 family, but ACTG1 mutations are not frequent among families with hereditary hearing impairment. Eur J Hum Genet 2006, 14(10):1097-1105.

32. Baek Jl, Oh SK, Kim DB, Choi SY, Kim UK, Lee KY, Lee SH: Targeted massive parallel sequencing: the effective detection of novel causative mutations associated with hearing loss in small families. Orphanet J Rare Dis 2012, 7(1):60.

33. de Heer AM, Huygen PL, Collin RW, Oostrik J, Kremer H, Cremers CW: Audiometric and vestibular features in a second Dutch DFNA20/26 family with a novel mutation in ACTG1. Ann Otol Rhinol Laryngol 2009, 118(5):382-390,

34. Liu P, Li H, Ren X, Mao H, Zhu Q, Zhu Z, Yang R, Yuan W, Liu J, Wang Q, et al: Novel ACTG1 mutation causing autosomal dominant non-syndromic hearing impairment in a Chinese family. J Genet Genomics 2008, 35(9):553-558.

35. Khaitlina SY: Functional specificity of actin isoforms. Int Rev Cytol 2001, 202:35-98.

36. Cho TJ, Kim OH, Lee HR, Shin SJ, Yoo WJ, Park WY, Park SS, Cho SI, Choi IH: Autosomal recessive multiple epiphyseal dysplasia in a Korean girl caused by novel compound heterozygous mutations in the DTDST (SLC26A2) gene. J Korean Med Sci 2010, 25(7):1105-1108.

37. Vigeland MD: paramlink: Parametric linkage analysis in $\mathrm{R}$. In $R$ package version 06-1. 2012. http://CRAN.R-project.org/package=paramlink.

38. Berman HM, Westbrook J, Feng Z, Gilliland G, Bhat TN, Weissig H, Shindyalov IN, Bourne PE: The Protein Data Bank. Nucleic Acids Res 2000 28(1):235-242.

39. Otterbein $L R$, Graceffa P, Dominguez R: The crystal structure of uncomplexed actin in the ADP state. Science 2001, 293(5530):708-711.

40. Spjuth O, Helmus T, Willighagen EL, Kuhn S, Eklund M, Wagener J, MurrayRust P, Steinbeck C, Wikberg JE: Bioclipse: an open source workbench for chemo- and bioinformatics. BMC Bioinforma 2007, 8:59.

doi:10.1186/1471-2164-14-191

Cite this article as: Park et al: Multiphasic analysis of whole exome sequencing data identifies a novel mutation of ACTG1 in a nonsyndromic hearing loss family. BMC Genomics 2013 14:191.

\section{Submit your next manuscript to BioMed Central and take full advantage of:}

- Convenient online submission

- Thorough peer review

- No space constraints or color figure charges

- Immediate publication on acceptance

- Inclusion in PubMed, CAS, Scopus and Google Scholar

- Research which is freely available for redistribution

Submit your manuscript at www.biomedcentral.com/submit 